\title{
Crosstalk analysis of pathways in breast cancer using a network model based on overlapping differentially expressed genes
}

\author{
YONG SUN ${ }^{1,2 *}$, KAI YUAN ${ }^{3 *}$, PENG ZHANG $^{2}$, RONG MA $^{4}$, QI-WEN ZHANG ${ }^{2}$ and XING-SONG TIAN ${ }^{1}$ \\ ${ }^{1}$ Department of Breast and Thyroid Surgery, Shandong Provincial Hospital, Shandong University, Jinan, Shandong 250021; \\ ${ }^{2}$ Department of General Surgery, Laiwu Hospital Affiliated to Taishan Medical College, Laiwu, Shandong 271100; \\ ${ }^{3}$ Department of Breast Surgery, Shandong Provincial Qianfoshan Hospital, Shandong University; \\ ${ }^{4}$ Department of Breast Surgery, Qilu Hospital, Shandong University, Jinan, Shandong 250014, P.R. China
}

Received August 14, 2014; Accepted May 7, 2015

DOI: $10.3892 / \mathrm{etm} .2015 .2527$

\begin{abstract}
Multiple signal transduction pathways can affect each other considerably through crosstalk. However, the presence and extent of this phenomenon have not been rigorously studied. The aim of the present study was to identify strong and normal interactions between pathways in breast cancer and determine the main pathway. Five sets of breast cancer data were downloaded from the high-throughput Gene Expression Omnibus (GEO) and analyzed to identify differentially expressed (DE) genes using the Rank Product (RankProd) method. A list of pathways with differential expression was obtained by gene set enrichment analysis (GSEA) of the Kyoto Encyclopedia of Genes and Genomes (KEGG) database. The DE genes that overlapped between pathways were identified and a crosstalk network diagram based on the overlap of DE genes was constructed. A total of 1,464 DE genes and 26 pathways were identified. In addition, the number of DE genes that overlapped between specific pathways were determined, and the greatest degree of overlap was between the extracellular matrix (ECM)-receptor interaction and Focal adhesion pathways, which had 22 overlapping DE genes. Weighted pathway analysis of the crosstalk between pathways identified that Pathways in cancer was the main pathway in breast cancer.
\end{abstract}

\section{Introduction}

Breast cancer is a malignant type of tumor with the highest incidence in women, and it seriously affects the quality of life

Correspondence to: Dr Xing-Song Tian, Department of Breast and Thyroid Surgery, Shandong Provincial Hospital, Shandong University, 324 Weiqi Road, Jinan, Shandong 250021, P.R. China

E-mail: xingsongtian@yeah.net

* Contributed equally

Key words: crosstalk, RankProd, gene set enrichment analysis, breast cancer, pathway of patients (1). The occurrence of breast cancer is considered to be the result of the abnormal expression of numerous genes. The overexpression of a variety of oncogenes and tumor suppressor gene deletion, mutation or low expression accompany carcinogenesis $(2,3)$. However, the mechanism of the occurrence and development of breast cancer remains incompletely understood, and numerous genes and their functions remain to be discovered and understood. A certain theoretical basis for further study of the pathogenesis of breast cancer is provided by the screening of differentially expressed (DE) genes in breast cancer tissue and normal breast tissue and analyzing the pathways in which these genes are enriched using biological information $(4,5)$.

Experimental high-throughput genomics has identified a number of notable genes, including DE genes having significant changes in expression level. A main task in bioinformatics is to elucidate the biological significance of these genes. Based on a variety of biological knowledge databases such as the Gene Ontology (GO) database and the Kyoto Encyclopedia of Genes and Genomes (KEGG) pathway database, numerous research groups are systemically analyzing the biological processes and single pathways associated with the enriched genes using a variety of statistical analysis strategies. At present, studies have clearly shown that UBE2C (6,7), EGFR (8) and interferon regulatory factor binding protein 2 (IRF2BP2) (9) are $\mathrm{DE}$ genes associated with the development of breast cancer. Further study of these genes may be helpful in preventing the occurrence of this disease.

Pathways can affect each other through a phenomenon known as crosstalk, rather than acting independently (10). Although it is evident that different pathways could influence each other, particularly when there is an overlap of DE genes having significant changes in expression level, the presence and extent of this phenomenon have not been rigorously studied. Identification of the interaction of pathways has important implications for the understanding of numerous diseases; it may contribute to their prevention and treatment by enabling inhibition of the interaction among pathways, which may play a key role in the invasion and proliferation of cancer cells (11-13). Wang et al (14) showed that EGFR activity was increased in a PTGS2 (COX-2) transgenic mouse and that forced expression of PTGS2 (COX-2) in human colorectal 
cancer (CRC) cells stimulated cellular proliferation. In addition, it was demonstrated that a crosstalk of PTGS2 (COX-2) and EGFR pathways synergistically promoted CRC progression and metastasis. The study conducted by Krysan et al (15) indicated that PGE2 was able to induce the proliferation of colon and lung cancer cells through the activation of MAPK in an EGFR-independent manner in vitro. Wang et al (16) generated a mouse model characterized by the co-expression of activated forms of AKT and Ras in the liver. The results indicated that concomitant suppression of AKT/mTOR and Ras/MAPK pathways was highly detrimental for the growth of AKT/Ras-expressing cells in vitro. This finding has important implications for the understanding of HCC pathogenesis and its prevention.

The identification of DE genes and the pathways involved in the development of disease has been the subject of numerous studies. However, in many cases the crosstalk between pathways was not investigated, and the enriched DE genes and the most important pathway were not identified. In the present study, 5 sets of breast cancer data were downloaded from the Gene Expression Omnibus (GEO) platform and analyzed with the RankProd package (17) to detect DE genes. The pathways in which the DE genes were enriched were identified by the gene set enrichment analysis (GSEA) $(18,19)$ method. The DE genes that overlapped between pathways were identified for further analysis. A network diagram of crosstalk among these pathways was constructed based on the overlapping DE genes with the aim of identifying the main pathway on the basis of the connections with other pathways.

\section{Materials and methods}

Detection of differentially expressed genes. The study design was to obtain experimental data for breast cancer from a genomic database, and to identify DE genes and their pathways from the data using analytical software. The aim was to obtain an improved understanding of the interaction among pathways by analyzing the crosstalk of pathways.

Five biological data sets for breast cancer (E-GEOD-29431, E-GEOD-3744, E-GEOD-42568, E-GEOD-50567 and E-GEOD-7904) from different experimental origins were downloaded from the Gene Expression Omnibus (GEO) database (http://www.ncbi.nlm.nih.gov/geo/). There were 281 breast cancer samples and 69 normal samples in total. After pretreating these data by RAM, quantiles and median polish summarization methods, unqualified chips were eliminated leaving only qualified data to enter the next step. The gene expression values of all data were transformed to a comparable level, which provided a digital expression profile for subsequent analysis. As the 5 sets of data were from different experiments, DE genes were detected using RankProd (http://www.bioconductor.org/packages/release/bioc/html/RankProd.html), which is a powerful meta-analysis tool for integrating multiple array datasets from various experimental platforms. In this analysis, $\mathrm{T}$ and $\mathrm{C}$ represent two experimental conditions (treatment versus control), and there are $\mathrm{nT}$ and $\mathrm{nC}$ replicates in the first dataset, $\mathrm{mT}$ and $\mathrm{mC}$, $\mathrm{sT}$ and $\mathrm{sC}$, wT and $\mathrm{wC}$, and $\mathrm{fT}$ and $\mathrm{fC}$ replicates in the second, third, fourth and fifth data sets, respectively.
The rank product for each gene $\left(\mathrm{RP}_{\mathrm{g}}\right)$ was calculated using the following formula:

$$
R P_{g}=\left(\prod_{i} r_{g i}\right)^{1 / K}
$$

$r_{g i}$ is the rank of the gth gene under $i$ th comparison. $i=1, \ldots, \mathrm{K}$, where $\mathrm{K}=(\mathrm{nT} \times \mathrm{nC})+(\mathrm{mT} \times \mathrm{mC})+(\mathrm{sT} \times \mathrm{sC})+(\mathrm{wT} \times \mathrm{wC})$ $+(\mathrm{fT} \times \mathrm{fC})$. The genes with $\mid \log \mathrm{FCl}>2$ and percentage of false predictions $(\mathrm{pfp})<0.01$ were considered differentially expressed.

GSEA. Following the detection of DE genes from the 5 breast cancer data sets, the next step was to find pathways in which DE genes were enriched using gene enrichment analysis. There are three types of common gene enrichment analysis: singular enrichment analysis (SEA), GSEA and modular enrichment analysis (MEA). In this study, a broader application of the GSEA (20) analysis method was used. The prior knowledge of biology such as that available from KEGG (http://www. genome.jp/kegg/) was used to identify a set of genomes. Each genome was given an enrichment score (ES) by statistical calculation, the difference in significance level of the ES for different groups was detected (i.e., breast cancer and normal), then the significance level was adjusted to $\mathrm{P}<0.05$ to obtain a list of enriched gene pathways.

The ES was calculated as follows:

$$
\begin{gathered}
E S(S)=\max _{1 \leq i \leq N}|\operatorname{Hit}(S, i)-\operatorname{Miss}(S, i)| \\
\left.\operatorname{Hit}(S, i)=\left.\sum_{\substack{g_{i} \subseteq S \\
1 \leq j \leq i}}|| r_{i}\right|^{\mathrm{p}} / \sum_{j \in S}\left|r_{j}\right|^{\mathrm{p}}\right] \\
\operatorname{Miss}(S, i)=\sum_{\substack{g_{i} \not \subset s \\
1 \leq j \leq i}} \frac{1}{N-M}
\end{gathered}
$$

$S$ indicates the biological pathway, $r_{j}$ is the correlation of gene and phenotype, $M$ is the number of genes in $S$ and $N$ is the total number of DE genes in the KEGG database. $\mathrm{P}$ is used to correct the $E S$; it avoids erroneous inferences when the gene is located in the middle of gene set, as they would otherwise yield high $\mathrm{ES}$ values. Hit $(S, i)$ indicates the total increase in the $E S$ for cases when gene $i$ belongs to $S$; $\operatorname{Miss}(S, i)$ indicates the total reduction in ES for cases when gene $i$ does not belong to $S$.

Pathway crosstalk network analysis. The interactions between pathways that were obtained by the GSEA method were analyzed. It was assumed that if DE genes overlapped between pathways, an interaction existed between the pathways. If the number of DE genes that overlapped between pathways was $>5$, the two pathways were considered to have a strong interaction. Under these conditions, a network diagram was designed, programmed and constructed in order to represent the crosstalk between pathways visually.

The formula used was as follows:

$$
D=\sum_{\substack{1 \leq i \leq n, 1 \leq j \leq n}} N_{O D}
$$

$D$ indicates the degree of interaction between pathways. $N_{O D}$ indicates the number of DE genes that are overlapped, $i$ and 
Table I. Significant pathways.

\begin{tabular}{|c|c|c|c|}
\hline KEGG ID & Term & Count & P-value \\
\hline 04512 & ECM-receptor interaction & 25 & $1.99 \mathrm{E}-07$ \\
\hline 04510 & Focal adhesion & 43 & $3.87 \mathrm{E}-07$ \\
\hline 05200 & Pathways in cancer & 55 & $2.95 \mathrm{E}-05$ \\
\hline 05144 & Malaria & 13 & $5.66 \mathrm{E}-04$ \\
\hline 03320 & PPAR signaling pathway & 16 & 7.59E-04 \\
\hline 04110 & Cell cycle & 23 & $1.69 \mathrm{E}-03$ \\
\hline 04115 & p53 signaling pathway & 14 & $5.14 \mathrm{E}-03$ \\
\hline 05222 & Small cell lung cancer & 16 & 8.33E-03 \\
\hline 05218 & Melanoma & 14 & $8.75 \mathrm{E}-03$ \\
\hline 00565 & Ether lipid metabolism & 8 & 8.89E-03 \\
\hline 04530 & Tight junction & 22 & 8.95E-03 \\
\hline 04270 & Vascular smooth muscle contraction & 19 & 9.89E-03 \\
\hline 04114 & Oocyte meiosis & 19 & $9.89 \mathrm{E}-03$ \\
\hline 04520 & Adherens junction & 14 & $9.91 \mathrm{E}-03$ \\
\hline 04350 & TGF- $\beta$ signaling pathway & 15 & $1.21 \mathrm{E}-02$ \\
\hline 05215 & Prostate cancer & 16 & $1.29 \mathrm{E}-02$ \\
\hline 04610 & Complement and coagulation cascades & 13 & $1.29 \mathrm{E}-02$ \\
\hline 04710 & Circadian rhythm - mammal & 6 & $1.37 \mathrm{E}-02$ \\
\hline 04914 & Progesterone-mediated oocyte maturation & 15 & $1.67 \mathrm{E}-02$ \\
\hline 04614 & Renin-angiotensin system & 5 & $2.12 \mathrm{E}-02$ \\
\hline 00982 & Drug metabolism - cytochrome P450 & 11 & $2.81 \mathrm{E}-02$ \\
\hline 00564 & Glycerophospholipid metabolism & 13 & $3.12 \mathrm{E}-02$ \\
\hline 04916 & Melanogenesis & 16 & $3.29 \mathrm{E}-02$ \\
\hline 05219 & Bladder cancer & 8 & 4.49E-02 \\
\hline 05221 & Acute myeloid leukemia & 10 & $4.60 \mathrm{E}-02$ \\
\hline 04360 & Axon guidance & 19 & 4.65E-02 \\
\hline
\end{tabular}

KEGG, Kyoto Encyclopedia of Genes and Genomes; ECM, extracellular matrix; PPAR, peroxisome proliferator-activated receptor; TGF, transforming growth factor.

$J$ represent DEGs in two different pathways. When $i=j, N_{O D}$ increases one; when $i \neq j, N_{O D}$ is unchanged. $\Sigma N_{O D} \geq 5$ indicates a strong connection, whereas $\Sigma N_{O D}<5$ indicates a normal connection.

The total number of DE genes in a pathway overlapping with another pathway is Psum; the pathway with the maximum number (MaxPsum) was considered to be the central pathway.

\section{Results}

Detection of DE genes between breast cancer and normal breast tissue. DE genes were identified between breast cancer and normal breast tissue. Genes that were upregulated and downrregulated, respectively, in breast cancer compared with normal tissue were identified. Analysis of the DE genes revealed that there were $1,464 \mathrm{DE}$ genes in total, including 1,038 upregulated genes and 426 downregulated genes that had an estimated pfp $<0.01$ and $\mid \log F C l>2$.

Pathwayenrichment analysis. The majority of the pathways that were identified by the GSEA method to be significant $(\mathrm{P}<0.05)$ were cancer-related signaling pathways (Table I), for example, Pathways in cancer, Prostate cancer, Small cell lung cancer, peroxisome proliferator-activated receptor (PPAR) signaling pathway and p53 signaling pathway. As can be seen in Table I, a total of 26 pathways were included following enrichment analysis and the majority of the DE genes, with a count of 55, were contained in Pathways in cancer. The significance level $(\mathrm{P}<0.001)$ of the top five pathways in the list suggests the high reliability of the enrichment analysis.

Pathway crosstalk network analysis. According to the overlap of DE genes between pathways, a program was designed to generate an overall network diagram that could describe the crosstalk of all 26 pathways (Fig. 1A). In addition, a scattergram of overlapping DE genes in the pathway crosstalk network was drawn (Fig. 1B). It showed that the distribution of overlapping DE genes followed a power law $\left(\mathrm{y}=\mathrm{ax}{ }^{\mathrm{b}}\right.$, where $\mathrm{a}=42.08$ and $\left.\mathrm{b}=-1.18\right)$. In addition, a network diagram for pathways with a high degree of crosstalk, that is, those which contained $>5$ overlapping DE genes was generated (Fig. 2) and the main pathway was identified. It was found that the network of pathways with strong crosstalk 

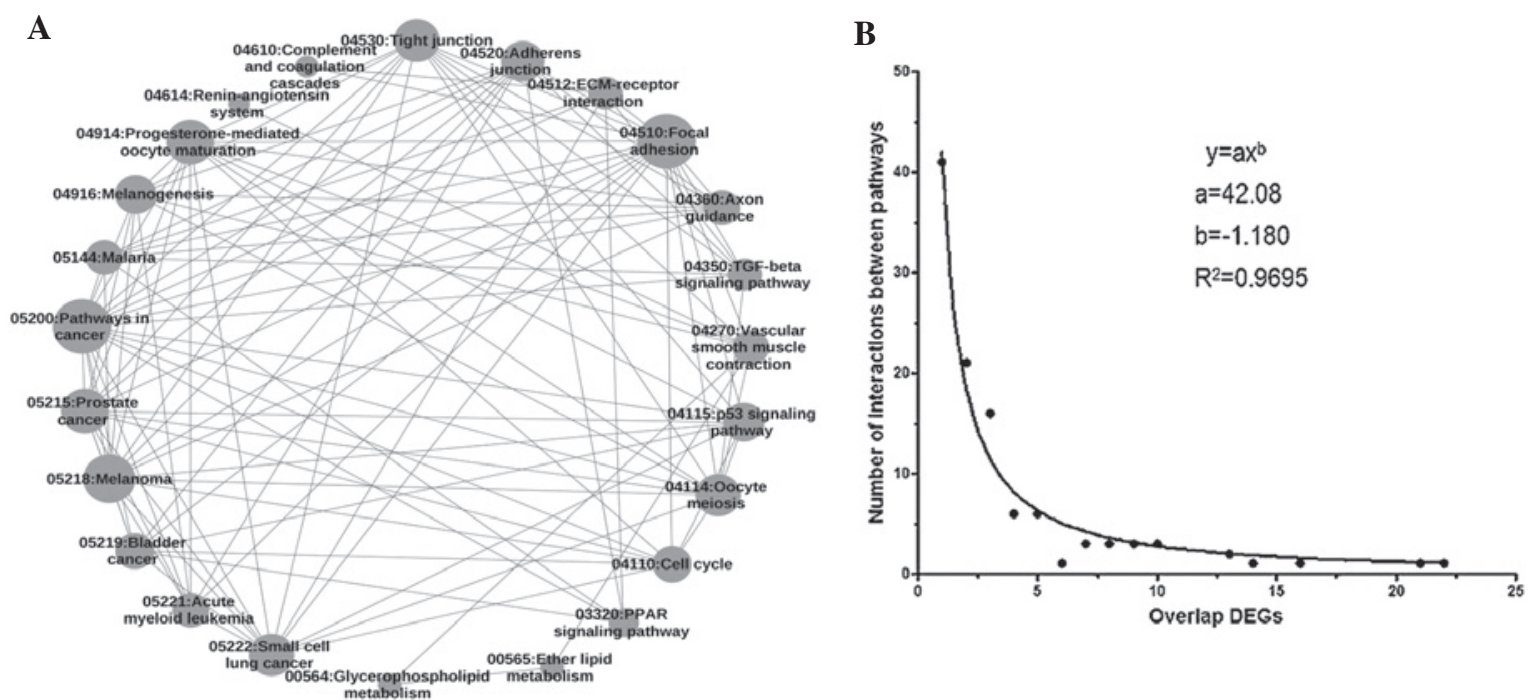

Figure 1. Interaction beween pathways based on the overlap of DE genes. (A) Network diagram of crosstalk among pathways; each circle represents a pathway, the size of the circles indicates how many DE genes are in the pathway; and the line between any two pathways indicates that there were DE genes that overlapped between them. (B) Scattergram of overlapping DE genes in the pathway crosstalk network. The network was a scale-free network whose distribution of overlapping DE genes followed a power law. DE, differentially expressed; DEGs, DE genes.

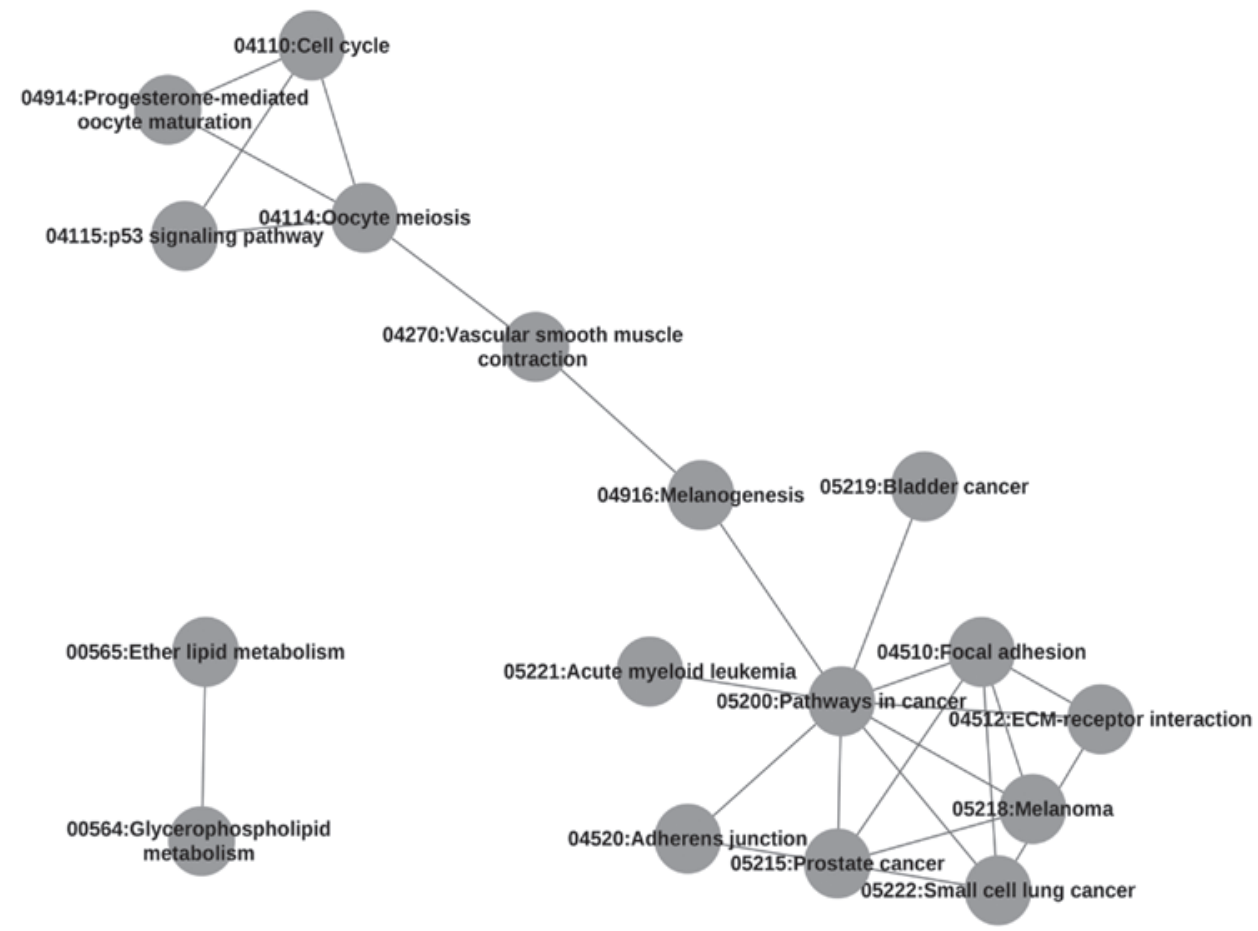

Figure 2. Network diagram of strong crosstalk among pathways. Each circle represents a pathway, and a line between circles indicates that there were $>5$ overlapping differentially expressed genes between the two pathways.

conformed to a scale-free network whose pathway degree distribution followed a power law $\left(\mathrm{y}=\mathrm{ax}{ }^{\mathrm{b}}\right.$, where $\mathrm{a}=43.48$ and $b=-0.5288$ ).

The pathways with the greatest number of DE genes overlapping between them were the extracellular matrix (ECM)-receptor interaction and Focal adhesion pathways, which had 22 overlapping DE genes. From the analytical results, it was found that Pathways in cancer, the most important pathway with a MaxPsum of 9 , had DE genes that overlapped with the Focal adhesion, Small cell lung cancer, Prostate cancer, Melanoma, ECM-receptor interaction, Acute myeloid leukemia, Melanogenesis, Adherens junction and Bladder cancer pathways (Table II). Furthermore, the DE genes that overlapped between the Pathways in cancer pathway and the other 9 pathways are listed in Table III.

\section{Discussion}

Overall, 1,464 DE genes were detected from 5 breast cancer data sets and 26 pathways were identified by the GSEA 
Table II. Differentially expressed genes that overlap between the main pathway (Pathways in cancer) and 9 other pathways.

\begin{tabular}{|c|c|c|c|}
\hline KEGG ID & Term & Overlap DEGs & Count \\
\hline 04510 & Focal adhesion & $\begin{array}{l}\text { IGF1, EGFR, LAMA2, PTEN, MAPK10, LAMA4, LAMC1, } \\
\text { MET, LAMA3, LAMB2, BCL2, PDGFA, COL4A6, FN1, } \\
\text { PIK3R1, PDGFRA, JUN, COL4A2, LAMB3, VEGFC, ITGA6 }\end{array}$ & 21 \\
\hline 05222 & Small cell lung cancer & $\begin{array}{l}\text { LAMA2, LAMA4, LAMC1, PTGS2, LAMB3, LAMA3, } \\
\text { LAMB2, BCL2, ITGA6, COL4A6, CCNE2, FN1, } \\
\text { E2F3, PTEN, COL4A2, PIK3R1 }\end{array}$ & 16 \\
\hline 05215 & Prostate cancer & $\begin{array}{l}\text { IGF1, FOXO1, TCF7L2, BCL2, FGFR1, EGFR, PDGFRA, } \\
\text { PDGFA, PTEN, TCF7L1, CCNE2, LEF1, E2F3, PIK3R1 }\end{array}$ & 14 \\
\hline 05218 & Melanoma & $\begin{array}{l}\text { IGF1, EGFR, FGF2, PTEN, FGF1, MET, PDGFRA, FGFR1, } \\
\text { PIK3R1, MITF, PDGFA, E2F3, CDKN2A }\end{array}$ & 13 \\
\hline 04512 & ECM-receptor interaction & $\begin{array}{l}\text { LAMA2, COL4A2, LAMA4, LAMC1, LAMB3, LAMA3, } \\
\text { LAMB2, ITGA6, COL4A6, FN1 }\end{array}$ & 10 \\
\hline 05221 & Acute myeloid leukemia & $\begin{array}{l}\text { ZBTB16, CEBPA, RUNX1T1, TCF7L2, STAT5A, STAT5B, } \\
\text { TCF7L1, KIT, PIK3R1, LEF1 }\end{array}$ & 10 \\
\hline 04916 & Melanogenesis & FZD4, TCF7L2, FZD5, TCF7L1, KIT, MITF, FZD7, LEF1 & 8 \\
\hline 04520 & Adherens junction & EGFR, TCF7L2, TGFBR, MET, TCF7L1, FGFR1, LEF1 & 7 \\
\hline 05219 & Bladder cancer & EGFR, VEGFC, MMP1, MMP9, FGFR3, E2F3, CDKN2A & 7 \\
\hline
\end{tabular}

KEGG, Kyoto Encyclopedia of Genes and Genomes; ECM, extracellular matrix.

Table III. Degree of connection among pathways.

\begin{tabular}{lll}
\hline KEGG ID & \multicolumn{1}{c}{ Pathway name } & Psum \\
\hline 04270 & Vascular smooth & \\
& muscle contraction & 2 \\
00564 & Glycerophospholipid & \\
& metabolism & 1 \\
00565 & Ether lipid metabolism & 1 \\
04115 & p53 signaling pathway & 2 \\
05219 & Bladder cancer & 1 \\
04520 & Adherens junction & 2 \\
04916 & Melanogenesis & 2 \\
04914 & Progesterone-mediated & 2 \\
04114 & oocyte maturation & 4 \\
04110 & Oocyte meiosis & 3 \\
05221 & Cell cycle & 1 \\
05218 & Acute myeloid leukemia & 3 \\
05215 & Melanoma & 5 \\
05222 & Prostate cancer & 4 \\
05200 & Small cell lung cancer & 9 \\
04510 & Pathways in cancer & 5 \\
04512 & Focal adhesion & 3 \\
\hline
\end{tabular}

KEGG, Kyoto Encyclopedia of Genes and Genomes; ECM, extracellular matrix.

method. Network diagrams of normal and strong crosstalk between pathways were constructed and Pathways in cancer was identified as the main pathway.
The hypothesis that the occurrence of breast cancer is the result of the abnormal expression of numerous genes (2) was confirmed. It is urgently necessary to identify genes that undergo changes in expression level in the process of breast cancer development in order to prevent the occurrence and development of tumors. Although numerous studies of DE genes in breast cancer have been conducted, the results have not been uniform $(21,22)$. The methods for detecting DE genes from microarray data are the significance analysis of microarrays (SAM) method (23), two sample t-test (24), Bonferroni correction and the Benjamini and Hochberg method (25). In the present study, the RankProd method was applied, which can analyze data sets of multiple origins simultaneously, and also offers several advantages including the biologically intuitive fold-change (FC) criterion, fewer assumptions under the model, and low numbers of replicates (26). A total of 1,464 DE genes were identified from the 5 experimental data sets through analysis. Among them, certain DE genes were consistent with those identified in previous studies, for instance, EGFR (8), BCL2 (27) and FN1 (28).

A broader application of the GSEA analysis method was used to conduct pathway enrichment analysis. The enrichment analysis strategy has two advantages: i) It reduces the impact of DE gene selection on the enrichment analysis; and ii) all the information from the chip experiments is used. The GSEA analysis identified 26 pathways enriched in DE genes, and the top ranked DE gene-enriched pathways were Pathways in cancer, Focal adhesion and ECM-receptor interaction. This finding was consistent a previous study in which certain pathways, such as Pathways in cancer and Cell cycle, were identified (29). Huan et al (29) determined the changes in metabolic pathways at different time points after the treatment of breast cancer samples with estradiol, using KEGG pathway enrichment analysis for the DE genes. They concluded that the 
changes were mainly focused on the Pathways in cancer, Focal adhesion, and Chemokine signaling pathways.

Due to the fast-growing human interactome knowledge base, network-based approaches have become increasingly powerful and informative for the study of disease mechanisms (30). Computational methods have been proposed for the detection of disease-related networks, including co-expression (31), protein-protein interaction (PPI) (32), protein phosphorylation (33) and DNA methylation (34) networks. To the best of our knowledge, no previous study has constructed a network based on overlapping DE genes and identified the most significant pathway in breast cancer using RankProd and GSEA methods. Furthermore, the present study showed overlapping genes in all pathways. Among them, EGFR (35), IGF-1 (36), E2F3 (37) are associated with lung cancer, prostate cancer and other diseases, which may be helpful for the study of diseases by understanding of these pathways. Although the crosstalk between pathways was analyzed and the main pathway was identified, further evaluation of how the pathways effect each other would be worthwhile. In addition, as high-throughput genomic technologies become more affordable and accurate, their use is likely to become more prevalent in the identification of candidate genes in the future.

\section{References}

1. Igene H: Global health inequalities and breast cancer: An impending public health problem for developing countries. Breast J 14: 428-434, 2008

2. Chia S, Norris B, Speers C, et al: Human epidermal growth factor receptor 2 overexpression as a prognostic factor in a large tissue microarray series of node-negative breast cancers. J Clin Oncol 26: 5697-5704, 2008.

3. Planas-Silva MD, Bruggeman RD, Grenko RT and Smith JS: Overexpression of $\mathrm{c}-\mathrm{Myc}$ and $\mathrm{Bcl}-2$ during progression and distant metastasis of hormone-treated breast cancer. Exp Mol Pathol 82: 85-90, 2007.

4. Ota D, Mimori K, Yokobori T, et al: Identification of recurrence-related microRNAs in the bone marrow of breast cancer patients. Int J Oncol 38: 955-962, 2011.

5. Ohira M and Nakagawara A: Global genomic and RNA profiles for novel risk stratification of neuroblastoma. Cancer Sci 101 2295-2301, 2010.

6. Fujita T, Ikeda H, Kawasaki K, Taira N, Ogasawara Y, Nakagawara A and Doihara H: Clinicopathological relevance of UbcH10 in breast cancer. Cancer Sci 100: 238-248, 2009.

7. Berlingieri MT, Pallante P, Sboner A, et al: UbcH10 is overexpressed in malignant breast carcinomas. Eur J Cancer 43: 2729-2735, 2007.

8. Wang S, Chen C, Meng Y, et al: Effective suppression of breast tumor growth by an anti-EGFR/ErbB2 bispecific antibody. Cancer Lett 325: 214-219, 2012.

9. Tinnikov AA, Yeung KT, Das S and Samuels HH: Identification of a novel pathway that selectively modulates apoptosis of breast cancer cells. Cancer Res 69: 1375-1382, 2009.

10. Donato M, Xu Z, Tomoiaga A, et al: Analysis and correction of crosstalk effects in pathway analysis. Genome Res 23: 1885-1893, 2013.

11. Bradley EW, Ruan MM, Vrable A and Oursler MJ: Pathway crosstalk between $\mathrm{Ras} / \mathrm{Raf}$ and $\mathrm{PI} 3 \mathrm{~K}$ in promotion of M-CSF-induced MEK/ERK-mediated osteoclast survival. J Cell Biochem 104: 1439-1451, 2008.

12. Gerits N, Kostenko S, Shiryaev A, Johannessen M and Moens U: Relations between the mitogen-activated protein kinase and the cAMP-dependent protein kinase pathways: Comradeship and hostility. Cell Signal 20: 1592-1607, 2008.

13. Pham H, Chong B, Vincenti R and Slice LW: Ang II and EGF synergistically induce COX-2 expression via CREB in intestinal epithelial cells. J Cell Physiol 214: 96-109, 2008.

14. Wang D, Xia D and Dubois RN: The crosstalk of PTGS2 and EGF signaling pathways in colorectal cancer. Cancers (Basel) 3: 3894-3908, 2011.
15. Krysan K, Reckamp KL, Dalwadi H, Sharma S, Rozengurt E, Dohadwala M and Dubinett SM: Prostaglandin E2 activates mitogen-activated protein kinase/Erk pathway signaling and cell proliferation in non-small cell lung cancer cells in an epidermal growth factor receptor-independent manner. Cancer Res 65: 6275-6281, 2005

16. Wang C, Cigliano A, Delogu S, Armbruster J, et al: Functional crosstalk between AKT/mTOR and Ras/MAPK pathways in hepatocarcinogenesis: Implications for the treatment of human liver cancer. Cell Cycle 12: 1999-2010, 2013.

17. Hong FX, Breitling R, McEntee CW, Wittner BS, Nemhauser JL and Chory J: A bioconductor package for detecting differentially expressed genes in meta-analysis. Bioinformatics 22: 2825-2827, 2006.

18. Mootha VK, Lindgren CM, Eriksson KF, et al: PGC-1alpha-responsive genes involved in oxidative phosphorylation are coordinately downregulated in human diabetes. Nat Genet 34: 267-273, 2003.

19. Suárez-Fariñas M, Lowes MA, Zaba LC and Krueger JG: Evaluation of the psoriasis transcriptome across different studies by gene set enrichment analysis (GSEA). PLoS ONE 5: e10247, 2010.

20. Subramanian A, Tamayo P, Mootha VK, et al: Gene set enrichment analysis: A knowledge-based approach for interpreting genome-wide expression profiles. Proc Natl Acad Sci USA 102: 15545-15550, 2005.

21. Lisowska KM, Dudaladava V, Jarzab M, et al: BRCA1-related gene signature in breast cancer: The role of ER status and molecular type. Front Biosci (Elite Ed) 3: 125-136, 2011.

22. Richardson AL, Wang ZC, De Nicolo A, et al: X chromosomal abnormalities in basal-like human breast cancer. Cancer Cell 9: 121-132, 2006.

23. Efron B and Tibshirani R: Empirical Bayes methods and false discovery rates for microarrays. Genet Epidemiol 23: 70-86, 2002.

24. Cui X and Churchill GA: Statistical tests for differential expression in cDNA microarray experiments. Genome Biol 4: 210, 2003.

25. Yekutieli D and Benjamini Y: Resampling-based false discovery rate controlling multiple test procedures for correlated test statistics. J Stat Plan Inference 82: 171-196, 1999.

26. Breitling $R$ and Herzyk P: Rank-based methods as a non-parametric alternative of the T-statistic for the analysis of biological microarray data. J Bioinform Comput Biol 3: 1171-1189, 2005.

27. Long JM, Bell CW, Fagg WS IV, et al: Microarray and pathway analysis reveals decreased CDC25A and increased CDC42 associated with slow growth of BCL2 overexpressing immortalized breast cell line. Cell Cycle 7: 3062-3073, 2008.

28. Bissell MJ, Radisky DC, Rizki A, Weaver VM and Petersen OW: The organizing principle: Microenvironmental influences in the normal and malignant breast. Differentiation 70: 537-546, 2002.

29. Huan J, Wang L, Xing L, et al: Insights into significant pathways and gene interaction networks underlying breast cancer cell line MCF-7 treated with 17ß-estradiol (E2). Gene 533: 346-355, 2014.

30. del Sol A, Balling R, Hood L and Galas D: Diseases as network perturbations. Curr Opin Biotechnol 21: 566-571, 2010.

31. Miller JA, Oldham MC and Geschwind DH: A systems level analysis of transcriptional changes in Alzheimer's disease and normal aging. J Neurosci 28: 1410-1420, 2008.

32. Shao L, Wang L, Wei Z, et al: Dynamic network of transcription and pathway crosstalk to reveal molecular mechanism of MGd-treated human lung cancer cells. PLoS ONE 7: e31984, 2012.

33. Tian M, Chen X, Xiong Q, et al: Phosphoproteomic analysis of protein phosphorylation networks in Tetrahymena thermophila, a model single-celled organism. Mol Cell Proteomics 13: 503-519, 2014.

34. Bartlett TE, Olhede SC and Zaikin A: A DNA methylation network interaction measure, and detection of network oncomarkers. PLoS ONE 9: e84573, 2014.

35. Remon J, Morán T, Reguart N, Majem M, Carcereny E and Lianes P: Beyond EGFR TKI in EGFR-mutant non-small cell lung cancer patients: Main challenges still to be overcome. Cancer Treat Rev 40: 723-729, 2014.

36. Wang Z, Wang Z, Liang Z, et al: Expression and clinical significance of IGF-1, IGFBP-3, and IGFBP-7 in serum and lung cancer tissues from patients with non-small cell lung cancer. Onco Targets Ther 6: 1437-1444, 2013.

37. Bilke S, Schwentner R, Yang F, et al: Oncogenic ETS fusions deregulate E2F3 target genes in Ewing sarcoma and prostate cancer. Genome Res 23: 1797-1809, 2013. 